QUARTERLY OF APPLIED MATHEMATICS

VOLUME LXIII, NUMBER 3

SEPTEMBER 2005, PAGES 429-453

S 0033-569X(05)00967-4

Article electronically published on July 11, 2005

\title{
STABILIZATION OF A SYSTEM OF ANISOTROPIC THERMOELASTICITY BY NONLINEAR BOUNDARY AND INTERNAL FEEDBACKS
}

\author{
BY \\ AMAR HEMinNA (U.S.T.H.B., Fac de Maths, El-Alia, Bab Ezzouar, Alger Algérie), \\ SERGE NICAISE (Université de Valenciennes et du Hainaut-Cambrésis, MACS, Institut des \\ Sciences et Techniques de Valenciennes, F-59313 Valenciennes Cedex 9, France), \\ AND \\ ABDOUlAYE SÈnE (Université Cheikh Anta DIOP, Département de Mathématiques, Faculté des \\ sciences et techniques, Dakar Sénégal)
}

Abstract. We consider the stabilization of an anisotropic thermoelasticity system with a natural Neumann boundary condition on part of the boundary and combined nonlinear internal and boundary feedbacks. We then give an answer to a problem raised by Liu and Zuazua.

1. Introduction. Let $\Omega$ be a nonempty bounded open subset of $\mathbb{R}^{n}, n \geq 1$, with a boundary $\Gamma$ of class $C^{2}$. We denote by $\nu=\left(\nu_{1}, \cdots, \nu_{n}\right)^{\top}$ the unit outward normal vector along $\Gamma$. For a fixed $x_{0} \in \mathbb{R}^{n}$ we define the function $m(x)=x-x_{0} ; x \in \mathbb{R}^{n}$ and the following partition of the boundary $\Gamma$ (see Figure 1):

$$
\begin{aligned}
& \Gamma_{1}=\{x \in \Gamma: m(x) \cdot \nu(x) \leq 0\}, \\
& \Gamma_{2}=\{x \in \Gamma: m(x) \cdot \nu(x)>0\} .
\end{aligned}
$$

In this paper we consider the system of anisotropic thermoelasticity:

$$
\begin{cases}u^{\prime \prime}-\operatorname{div} \sigma(u)+\alpha \nabla \theta+f\left(u^{\prime}\right) & =0 \text { in } Q:=\Omega \times \mathbb{R}^{+}, \\ \theta^{\prime}-\Delta \theta+\beta \operatorname{div} u^{\prime} & =0 \text { in } Q, \\ \theta & =0 \text { on } \Sigma=\Gamma \times \mathbb{R}^{+}, \\ u & =0 \text { on } \Sigma_{1}=\Gamma_{1} \times \mathbb{R}^{+}, \\ \sigma(u) \cdot \nu+a u+g\left(u^{\prime}\right) & =0 \text { on } \Sigma_{2}=\Gamma_{2} \times \mathbb{R}^{+}, \\ u(\cdot, 0)=u_{0}, u^{\prime}(\cdot, 0)=u_{1} \theta(\cdot, 0)=\theta_{0} & \text { in } \Omega,\end{cases}
$$

Received February 1, 2004.

2000 Mathematics Subject Classification. Primary 35B35, 73B30.

E-mail address: ahemina@hotmail.com

E-mail address: s.nicaise@univ-valenciennes.fr

E-mail address: abdousen@ucad.sn 
where $u=u(x, t)=\left(u_{1}(x, t), \cdots, u_{n}(x, t)\right)^{\top}$ denotes the displacement vector field, $\theta=\theta(x, t)$ the temperature and the stress tensor $\sigma$ is defined by $\sigma_{i j}(u)=a_{i j k l} \varepsilon_{k l}(u)$ (throughout the paper we adopt the convention of repeated indices), where $\varepsilon(u)$ is the strain tensor given by

$$
\varepsilon_{i j}(u)=\frac{1}{2}\left(\partial_{j} u_{i}+\partial_{i} u_{j}\right)
$$

$\left(\partial_{i}=\frac{\partial}{\partial x_{i}}\right)$ and the coefficients $a_{i j k l}$ of class $C^{2}(\bar{\Omega})$ are such that

$$
a_{i j k l}=a_{k l i j}=a_{j i k l}
$$

and satisfy the ellipticity condition:

$$
\exists \delta>0: a_{i j k l} \varepsilon_{i j} \varepsilon_{k l} \geq \delta \varepsilon_{i j} \varepsilon_{i j},
$$

for all symmetric tensor $\varepsilon_{i j}$.

The components of the vector field $\operatorname{div} \sigma(u)$ are given by

$$
(\operatorname{div} \sigma(u))_{i}=\partial_{j} \sigma_{i j}, i=1, \ldots, n .
$$

The function $a$ is nonnegative and belongs to $C^{1}\left(\Gamma_{2}\right)$; the functions $g(u)=\left(g_{1}(u), \ldots\right.$, $\left.g_{n}(u)\right)^{\top}$ and $f(u)=\left(f_{1}(u), \ldots, f_{n}(u)\right)^{\top}$ are continuous and satisfy

$$
\begin{aligned}
& f(0)=g(0)=0, \\
& (g(x)-g(y)) \cdot(x-y) \geq 0, \forall x, y \in \mathbb{R}^{n}, \\
& (f(x)-f(y)) \cdot(x-y) \geq 0, \forall x, y \in \mathbb{R}^{n} .
\end{aligned}
$$

The coupling parameters $\alpha$ and $\beta$ are supposed to be positive.

These assumptions guarantee that the system (3) is dissipative since its energy defined by

$$
E(t)=\frac{1}{2} \int_{\Omega}\left\{\left|u^{\prime}\right|^{2}+\sigma(u): \varepsilon(u)+\frac{\alpha}{\beta}|\theta|^{2}\right\} d x+\frac{1}{2} \int_{\Gamma_{2}} a|u|^{2} d \Gamma
$$

is nonincreasing.

The stabilization of this system without feedback has been analyzed in 14 and cannot be guaranteed for all domains since the damping produced by the heat equation is not sufficient. Therefore, it is natural to study the stabilization of this system by adding supplemantary damping terms. According to the existing literature on damping, both internal or boundary damping are natural ways of doing that.

The stabilization of different variants of the system (3) has been studied in the literature, notably in [15, 16] (see also [5, 17, 18, 19, 20]) in the isotropic case but with nonnatural boundary conditions, namely by replacing in (3) the term $\sigma(u) \cdot \nu$ by $\mu \frac{\partial u}{\partial n}+(\lambda+\mu) \nu \operatorname{div} u$. In [15], Liu considered the case $f=0$ and the linear feedback, i.e., $g(x)=x$. Recently, Liu and Zuazua [16] have established, still in the case $f=0$, exponential, polynomial or logarithmic decay of the energy for some nonlinearities $g$. The aim of this work is to generalize these results to the case of an anisotropic system, to the case $f \neq 0$ and to the case of natural boundary conditions. This last case answers a question raised in [16. For this purpose, in the linear case we establish integral inequalities as in [15] and 1] leading to the exponential decay and in the nonlinear case, we use the theorical results established in [13. In fact, the results from 13 guarantee that, if the linear system has an exponential decay, then the nonlinear system is automatically stable, the 


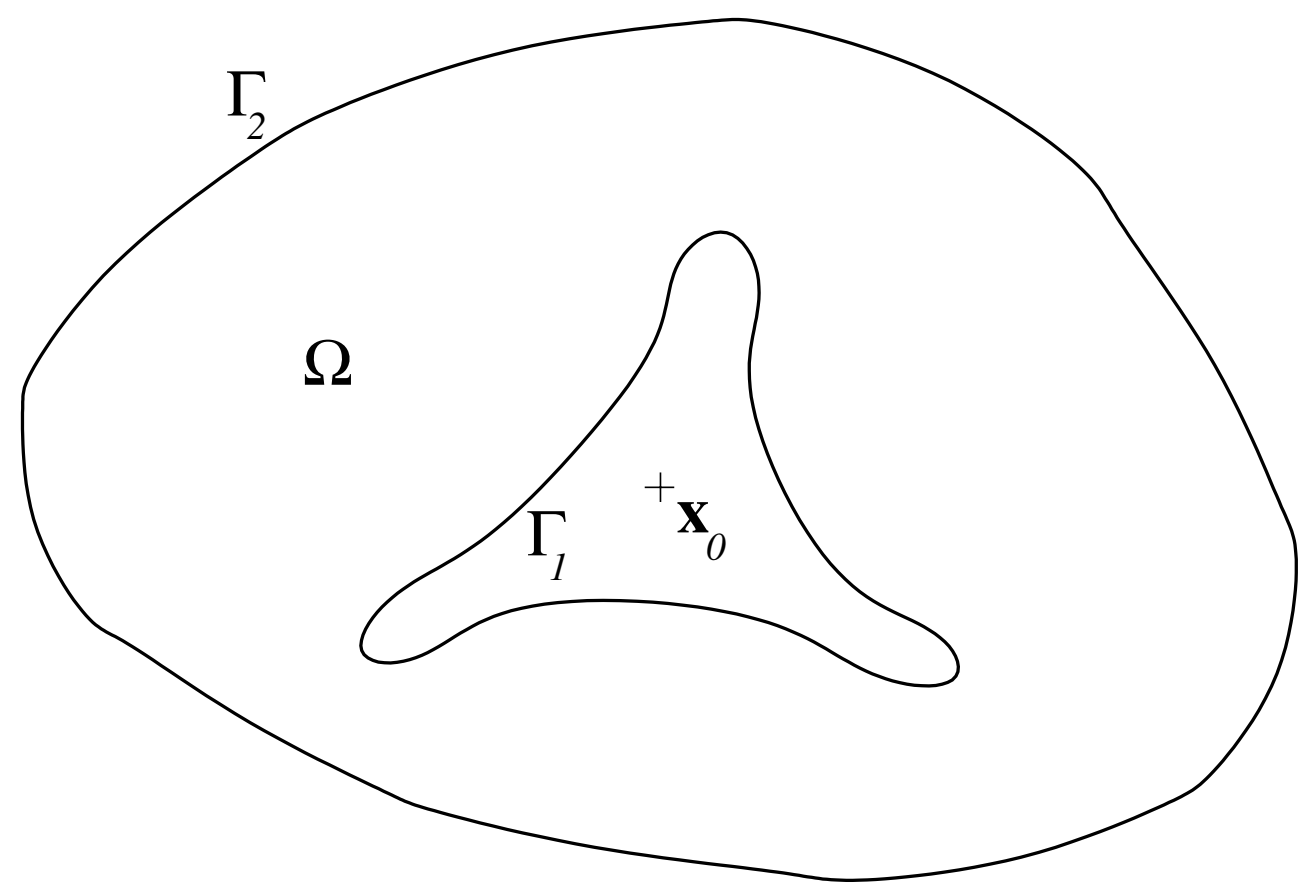

FIG. 1. An example of an open set $\Omega$.

decay rate being related to the properties of the nonlinearity. The main idea from 13 , is to use Liu's principle in a abstract setting and an appropriate integral inequality.

The results of this paper have been announced in [6] in the case of constant coefficients $a_{i j k l}$.

2. The main results. In the remainder of our paper we suppose that

$$
\Gamma_{1} \neq \emptyset \text { or } a(x)>0, \forall x \in \Gamma_{2} \text {. }
$$

Furthermore, in order to avoid regularity problems related to the change of boundary conditions we assume that (see Figure 1)

$$
\overline{\Gamma_{1}} \cap \overline{\Gamma_{2}}=\emptyset .
$$

Since we impose Dirichlet boundary conditions on the temperature $\theta$ and mixed boundary conditions on the displacement $u$, the regularity problem of the solution to our system is related to the one of the elastodynamic system. We then refer to 2 for the treatment of the case $\overline{\Gamma_{1}} \cap \overline{\Gamma_{2}} \neq \emptyset$ (in the case of 2D-polygonal domains).

We finally suppose that there exist positive constants $C_{f}$ and $C_{g}$ such that

$$
\begin{array}{r}
|f(x)| \leq C_{f}(1+|x|), \quad \forall x \in \mathbb{R}^{n}, \\
|g(x)| \leq C_{g}(1+|x|), \quad \forall x \in \mathbb{R}^{n} .
\end{array}
$$


We define the following Hilbert spaces:

$$
\begin{aligned}
H_{\Gamma_{1}}^{1}(\Omega) & =\left\{u \in H^{1}(\Omega) ; u=0 \text { on } \Gamma_{1}\right\} \\
W & =\left(H_{\Gamma_{1}}^{1}(\Omega)\right)^{n} \times\left(L^{2}(\Omega)\right)^{n} \\
\mathcal{H} & =W \times L^{2}(\Omega)
\end{aligned}
$$

The space $W$ is equipped with the natural norm:

$$
\|(u, v)\|_{W}^{2}=\int_{\Omega}\left[|v|^{2}+\sigma(u): \varepsilon(u)\right] d x+\int_{\Gamma_{2}} a|u|^{2} d \Gamma,
$$

where $\sigma(u): \varepsilon(u)=\sigma_{i j}(u) \varepsilon_{i j}(u)$.

In the sequel, we denote by $\langle\cdot, \cdot\rangle$ the duality pairing between $H_{0}^{1}(\Omega)$ and $H^{-1}(\Omega)$, or between $\left(H_{\Gamma_{1}}^{1}(\Omega)\right)^{n}$ and $\left[\left(H_{\Gamma_{1}}^{1}(\Omega)\right)^{n}\right]^{\prime}$, and by $(\cdot, \cdot)$ the inner product in $\left(L^{2}(\Omega)\right)^{n}$.

Theorem 2.1. Let $\Gamma_{1}$ and $\Gamma_{2}$ be defined by (11) and (2) and satisfying (9) and (10). Assume that the functions $f$ and $g$ satisfy (5), (6), (7), (11) and (12). Then for initial data $\left(u_{0}, u_{1}, \theta_{0}\right) \in \mathcal{H}$, the system (3) has a unique (weak) solution $(u, \theta)$ satisfying

$$
\left(u, u^{\prime}, \theta\right) \in C([0, \infty) ; \mathcal{H})
$$

We assume that there exists $\left.\gamma_{m} \in\right] 0,2[$ such that

$$
\left|m_{p} \partial_{p}\left(a_{i j k l}\right) \varepsilon_{i j} \varepsilon_{k l}\right| \leq \gamma_{m} a_{i j k l} \varepsilon_{i j} \varepsilon_{k l},
$$

for all symmetric tensor $\varepsilon_{i j}$.

The main result of our paper is the next theorem:

Theorem 2.2. Let $\Gamma_{1}$ and $\Gamma_{2}$ be given by (11) and (2) and satisfying (9) and (10). Assume that the coefficients $a_{i j k l}$ satisfy (4) and (14). Assume that the functions $f$ and $g$ satisfy (5), (6), (7), (11), (12), as well as the inequalities

$$
\begin{aligned}
& g(x) \cdot x \geq d|x|^{2} \forall x \in \mathbb{R}^{n}|x| \geq 1, \\
& |x|^{2}+|g(x)|^{2} \leq G(g(x) \cdot x) \forall x \in \mathbb{R}^{n}|x| \leq 1, \\
& |x|^{2}+|f(x)|^{2} \leq G(f(x) \cdot x) \forall x \in \mathbb{R}^{n}|x| \leq 1,
\end{aligned}
$$

where $d$ is a positive constant and $G$ a concave function defined on $\mathbb{R}_{+}$such that $G(0)=0$. Then there exist positive constants $\tau, r_{1}, r_{2}$ and a time $T_{1}>0$ (depending on $\left.\tau, E(0),\left|\Gamma_{2}\right|,|\Omega|\right)$ such that the energy of any solution of (3) satisfies

$$
E(t) \leq r_{2} G\left(\frac{\Psi^{-1}\left(r_{1} t\right)}{r_{1} \tau t}\right), \forall t \geq T_{1},
$$

where $\Psi$ is given by

$$
\Psi(t)=\int_{t}^{+\infty} \frac{1}{\Phi(s)} d s, \text { with } \Phi(s)=\tau R_{1} G^{-1}\left(\frac{s}{r_{2}}\right),
$$

and $R_{1}=\min \left(\left|\Gamma_{2}\right| ;|\Omega|\right)$.

Explicit decays are presented in Section 6.

REMARK 2.1. The previous Theorem still holds if $f=0$ and $g$ satisfies the previous hypotheses (case of boundary feedback only) or conversely if $\Gamma_{2}=\emptyset$ and $f$ satisfies the previous hypotheses (case of internal feedback). 
3. Well-posedness of the problem. In this section we prove Theorem 2.1 by reducing the system (3) to a first order evolution equation.

Let us define the operators

$$
A:\left(H_{\Gamma_{1}}^{1}(\Omega)\right)^{n} \longmapsto\left[\left(H_{\Gamma_{1}}^{1}(\Omega)\right)^{n}\right]^{\prime}
$$

and

$$
A_{0}: H_{0}^{1}(\Omega) \longmapsto H^{-1}(\Omega)
$$

by

$$
\begin{aligned}
\langle A u, v\rangle & =\int_{\Omega} \sigma(u): \varepsilon(v) d x, \quad \forall u, v \in\left(H_{\Gamma_{1}}^{1}(\Omega)\right)^{n}, \\
\left\langle A_{0} u, v\right\rangle & =\int_{\Omega} \nabla u \cdot \nabla v d x, \quad \forall u, v \in H_{0}^{1}(\Omega) .
\end{aligned}
$$

We further introduce the nonlinear operator $B_{0}$ from $\left(H_{\Gamma_{1}}^{1}(\Omega)\right)^{n}$ in $\left[\left(H_{\Gamma_{1}}^{1}(\Omega)\right)^{n}\right]^{\prime}$ by

$$
\left\langle B_{0} u, v\right\rangle=\int_{\Gamma_{2}} g(u) \cdot v d \Gamma+\int_{\Omega} f(u) \cdot v d x, \forall u, v \in\left(H_{\Gamma_{1}}^{1}(\Omega)\right)^{n} .
$$

Lemma 3.1. If the functions $f$ and $g$ satisfy (11) and (12), then the operator $B_{0}$ is well defined.

The proof of this lemma is similar to the one of Lemma 3.1 of [16] (see also section 6 of 13$]$.

To obtain the abstract formulation of (3), we multiply the first identity of the system (3) by $v \in\left(H_{\Gamma_{1}}^{1}(\Omega)\right)^{n}$ and we integrate by parts on $\Omega$, this yields

$$
\begin{aligned}
0 & =\int_{\Omega}\left[u^{\prime \prime}-\operatorname{div}(\sigma(u))+\alpha \nabla \theta+f\left(u^{\prime}\right)\right] \cdot v d x \\
& =\int_{\Omega} u^{\prime \prime} \cdot v d x-\int_{\Gamma}(\sigma(u) \cdot \nu) v d \Gamma+\int_{\Omega}[\sigma(u): \varepsilon(v)] d x \\
& +\int_{\Omega}(\alpha \nabla \theta \cdot v) d x+\int_{\Omega} f\left(u^{\prime}\right) \cdot v d x \\
& =\int_{\Omega} u^{\prime \prime} \cdot v d x+\int_{\Gamma} a \cdot u \cdot v d \Gamma+\int_{\Gamma} g\left(u^{\prime}\right) \cdot v d \Gamma \\
& +\int_{\Omega}[\sigma(u): \varepsilon(v)] d x+\int_{\Omega} \alpha \nabla \theta \cdot v d x+\int_{\Omega} f\left(u^{\prime}\right) \cdot v d x \\
& =\left\langle u^{\prime \prime}, v\right\rangle+\langle A u, v\rangle+\left\langle B_{0} u^{\prime}, v\right\rangle+\langle\alpha \nabla \theta, v\rangle .
\end{aligned}
$$

This leads to the identity

$$
u^{\prime \prime}+A u+B_{0} u^{\prime}+\alpha \nabla \theta=0 .
$$

In a similar manner, if we multiply the second identity of system (3) by $v \in\left(H_{\Gamma_{1}}^{1}(\Omega)\right)^{n}$ and if we integrate by parts on $\Omega$, we obtain

$$
\theta^{\prime}+A_{0} \theta+\beta \operatorname{div}\left(u^{\prime}\right)=0 .
$$

Setting

$$
\Phi=\left(u, u^{\prime}, \theta\right)
$$


and

$$
\mathcal{A} \Phi=\left(-u^{\prime}, A u+B_{0} u^{\prime}+\alpha \nabla \theta, A_{0} \theta+\beta \operatorname{div}\left(u^{\prime}\right)\right),
$$

the system (3) reduces to

$$
\left\{\begin{array}{l}
\Phi^{\prime}+\mathcal{A} \Phi=0 \\
\Phi(0)=\left(u_{0}, u_{1}, \theta_{0}\right) .
\end{array}\right.
$$

Lemma 3.2. Under the hypotheses (5), (6), (7), (9), (11) and (12), the operator $\mathcal{A}$ defined on $\mathcal{H}$ by (20), with domain

$$
D(\mathcal{A})=\left\{(u, v, \theta) \in \mathcal{H}: v \in\left(H_{\Gamma_{1}}^{1}(\Omega)\right)^{n}, A u+B_{0} v \in\left(L^{2}(\Omega)\right)^{n}, \theta \in H^{2}(\Omega) \cap H_{0}^{1}(\Omega)\right\}
$$

is maximal monotone. Moreover, $D(\mathcal{A})$ is dense in $\mathcal{H}$.

The proof of this lemma is similar to the one of Lemma 3.2 of [16]. The theory of nonlinear semi-groups [21] leads to Theorem 2.1]

4. An abstract stabilization result. In this section we recall the results obtained in [13. For brevity, we do not give the full proofs and refer to [13] for the details.

As mentioned in the introduction, the philosophy of [13] is that if the linear system has an exponential decay, then the nonlinear system is automatically stable, the decay rate being related to the properties of the nonlinearity. The two main steps are:

1. First we use Liu's principle in a abstract setting. This principle allows us to estimate the energy of the (nonlinear) direct system by terms relative to the inverse linear system with final condition equal to the initial condition of the direct system.

2. In a second step these terms are estimated using the exponential stability of the inverse linear system and an appropriate integral inequality.

We first describe a general abstract setting of hyperbolic type.

Let us fix two real separable Hilbert spaces $\mathcal{H}, \mathcal{V}$ with respective inner products $(., .)_{\mathcal{H}}$, $(.,.) \mathcal{V}$ and such that $\mathcal{V}$ is densely and continuously embedded into $\mathcal{H}$. Identifying $\mathcal{H}$ with its dual $\mathcal{H}^{\prime}$ we have the standard diagram:

$$
\mathcal{V} \hookrightarrow \mathcal{H}=\mathcal{H}^{\prime} \hookrightarrow \mathcal{V}^{\prime}
$$

We suppose given a bounded linear operator $A_{1}$ from $\mathcal{V}$ into $\mathcal{V}^{\prime}$ and a (nonlinear) mapping $B$ from $\mathcal{V}$ into $\mathcal{V}^{\prime}$. We now define two (nonlinear) operators $\mathcal{A}^{+}$and $\mathcal{A}^{-}$as follows:

$$
\begin{aligned}
& D\left(\mathcal{A}^{ \pm}\right)=\left\{v \in \mathcal{V} \mid\left( \pm A_{1}+B\right) v \in \mathcal{H}\right\}, \\
& \mathcal{A}^{ \pm}=\left( \pm A_{1}+B\right) v, \forall v \in D\left(\mathcal{A}^{ \pm}\right) .
\end{aligned}
$$

For brevity we often drop the superscript + from $\mathcal{A}^{+}$. 
Throughout this section we make the following assumptions:

$$
\begin{aligned}
& \mathcal{A}^{+} \text {is maximal monotone, } \\
& \mathcal{A}^{-} \text {is maximal monotone, } \\
& D\left(\mathcal{A}^{+}\right) \text {is dense in } \mathcal{H}, \\
& D\left(\mathcal{A}^{-}\right) \text {is dense in } \mathcal{H}, \\
& \left\langle A_{1} u, u\right\rangle=0, \forall u \in \mathcal{V}, \\
& \langle B u, u\rangle \geq 0, \forall u \in \mathcal{V},
\end{aligned}
$$

where here, above and below $\langle.,$.$\rangle means the duality pairing between \mathcal{V}^{\prime}$ and $\mathcal{V}$.

Using nonlinear semigroup theory [21, the evolution equation

$$
\left\{\begin{array}{l}
\frac{\partial u}{\partial t}+A_{1} u+B u=0 \text { in } \mathcal{H}, t \geq 0, \\
u(0)=u_{0},
\end{array}\right.
$$

admits a unique (weak) solution $u \in C\left(\mathbb{R}_{+}, \mathcal{H}\right)$ for any $u_{0} \in \mathcal{H}$. If, moreover, $u_{0} \in D(\mathcal{A})$, the problem (30) admits a unique (strong) solution $u \in W^{1, \infty}\left(\mathbb{R}_{+}, \mathcal{H}\right) \cap L^{\infty}\left(\mathbb{R}_{+}, D(\mathcal{A})\right)$ and such that $u(t) \in D(\mathcal{A})$, for all $t \geq 0$.

This system is dissipative since its energy

$$
\mathcal{E}(t)=\frac{1}{2}\|u(t)\|_{\mathcal{H}}^{2},
$$

is nonincreasing. Moreover, for $u_{0} \in D(\mathcal{A})$, we have

$$
\mathcal{E}(S)-\mathcal{E}(T)=\int_{S}^{T}\langle B u(t), u(t)\rangle d t, \forall 0 \leq S<T<\infty,
$$

The same results hold for $\mathcal{A}^{-}$(with the same expression for the energy and the same identity (32) for $u_{0} \in D\left(\mathcal{A}^{-}\right)$).

We first recall a necessary and sufficient condition which guarantees the exponential stability of (30). This condition is the validity of a stability estimate that will be checked in our particular case in the next section.

We start with the following definition.

Definition 4.1. We say that the pair $\left(A_{1}, B\right)$ satisfies the stability estimate if there exist $T>0$ and two nonnegative constants $C_{1}, C_{2}$ (which may depend on $T$ ) with $C_{1}<T$ such that

$$
\int_{0}^{T} \mathcal{E}(t) d t \leq C_{1} \mathcal{E}(0)+C_{2} \int_{0}^{T}\langle B u(t), u(t)\rangle d t,
$$

for all solutions $u$ of (30).

That property admits the following equivalent formulation:

Lemma 4.1. The pair $\left(A_{1}, B\right)$ satisfies the stability estimate if and only if there exist $T>0$ and a positive constant $C$ (which may depend on $T$ ) such that

$$
\mathcal{E}(T) \leq C \int_{0}^{T}\langle B u(t), u(t)\rangle d t
$$

for all solution $u$ of (30). 
Proof. $\Rightarrow$ : Since $\mathcal{E}(t)$ is nonincreasing, the estimate (33) implies that

$$
T \mathcal{E}(T) \leq \int_{0}^{T} \mathcal{E}(t) d t \leq C_{1} \mathcal{E}(0)+C_{2} \int_{0}^{T}\langle B u(t), u(t)\rangle d t .
$$

By the identity (32) we get

$$
T \mathcal{E}(T) \leq C_{1} \mathcal{E}(T)+\left(C_{1}+C_{2}\right) \int_{0}^{T}\langle B u(t), u(t)\rangle d t .
$$

This yields (34) with $C=\frac{C_{1}+C_{2}}{T-C_{1}}$.

$\Leftarrow$ : From the monotonicity of $\mathcal{E}$ we may write

$$
\int_{0}^{T} \mathcal{E}(t) d t \leq T \mathcal{E}(0)
$$

Again the identity (32) yields

$$
\int_{0}^{T} \mathcal{E}(t) d t \leq \frac{T}{2} \mathcal{E}(0)+\frac{T}{2}\left(\mathcal{E}(T)+\int_{0}^{T}\langle B u(t), u(t)\rangle d t\right) .
$$

Using the assumption (34) we obtain

$$
\left.\int_{0}^{T} \mathcal{E}(t) d t \leq \frac{T}{2} \mathcal{E}(0)+\frac{T}{2}(1+C) \int_{0}^{T}\langle B u(t), u(t)\rangle d t\right),
$$

which is nothing more than (33).

Let us now recall that Theorem 3.3 of [13] showed that the stability estimate is equivalent to the exponential stability of (30):

Theorem 4.1. The pair $\left(A_{1}, B\right)$ satisfies the stability estimate if and only if there exist two positive constants $M$ and $\omega$ such that

$$
\mathcal{E}(t) \leq M e^{-\omega t} \mathcal{E}(0),
$$

for all solutions $u$ of (30).

Proof. Assume that the stability estimate holds, i.e., by the previous lemma, (34) equivalently holds. The identity (32) then yields

$$
\mathcal{E}(T) \leq C(\mathcal{E}(0)-\mathcal{E}(T)) .
$$

This estimate is equivalent to

$$
\mathcal{E}(T) \leq \gamma \mathcal{E}(0),
$$

with $\gamma=\frac{C}{1+C}$ which is $<1$.

Applying this argument on $[(m-1) T, m T]$, for $m=1,2, \cdots$ (which is valid since our system is invariant by a translation in time), we will get

$$
\mathcal{E}(m T) \leq \gamma \mathcal{E}((m-1) T) \leq \cdots \leq \gamma^{m} \mathcal{E}(0), m=1,2, \cdots
$$

Therefore, we have

$$
\mathcal{E}(m T) \leq e^{-\omega m T} \mathcal{E}(0), m=1,2, \cdots
$$


with $\omega=\frac{1}{T} \ln \frac{1}{\gamma}>0$. For an arbitrary positive $t$, there exists $m=1,2, \cdots$ such that $(m-1) T<t \leq m T$ and by the nonincreasing property of $\mathcal{E}$, we conclude

$$
\mathcal{E}(t) \leq \mathcal{E}((m-1) T) \leq e^{-\omega(m-1) T} \mathcal{E}(0) \leq \frac{1}{\gamma} e^{-\omega t} \mathcal{E}(0) .
$$

The converse implication is based on the identity (32).

By Russell's principle the exponential stability lead to exact controllability results for the evolution equation associated with the operator $-A_{1}$ with controls in $L^{2}(] 0, T[; U)$, the control space $U$ being a given real Hilbert space such that $\mathcal{V}$ is continuously embedded into $U$. We then denote by $I_{U}$ the embedding from $\mathcal{V}$ into $U$ and $\mathcal{I}_{U}$ the mapping identifying $U$ as a subspace of $\mathcal{V}^{\prime}$, i.e.,

$$
\left\langle\mathcal{I}_{U} u, v\right\rangle:=\left(I_{U} u, I_{U} v\right)_{U}, \forall u, v \in \mathcal{V} .
$$

The exact controllability problem may be formulated as follows: for all $u_{0} \in \mathcal{H}$, we are looking for a time $T>0$ and a control $J \in L^{2}(] 0, T[; U)$ such that the solution $u$ of

$$
\left\{\begin{array}{l}
\frac{\partial u}{\partial t}-A_{1} u=J \text { in } \mathcal{V}^{\prime}, t \geq 0 \\
u(0)=u_{0}
\end{array}\right.
$$

satisfies

$$
u(T)=0
$$

We now recall Theorem 4.1 of [13]:

Theorem 4.2. If the assumptions (24) to (29) hold for the pair $\left(A_{1}, \mathcal{I}_{U}\right)$ and if the pair $\left(A_{1}, \mathcal{I}_{U}\right)$ satisfies the stability estimate, then for $T>0$ sufficiently large, for all $u_{0} \in \mathcal{H}$ there exist a control $J \in L^{2}(] 0, T[; U)$ such that the solution $u \in C([0, T], \mathcal{H})$ of (36) is at rest a time $T$, i.e., satisfies (37).

Proof. For further purposes we prefer to solve the inverse problem (so that the asumption " $\left(A_{1}, \mathcal{I}_{U}\right)$ satisfies the stability estimate" is replaced by " $\left(-A_{1}, \mathcal{I}_{U}\right)$ satisfies the stability estimate" ): Given $p_{0} \in \mathcal{H}$, we are looking for $K \in L^{2}(] 0, T[; U)$ such that the solution $p \in C([0, T], \mathcal{H})$ of

$$
\left\{\begin{array}{l}
\frac{\partial p}{\partial t}+A_{1} p=K \text { in } \mathcal{V}^{\prime}, t \geq 0 \\
p(T)=p_{0}
\end{array}\right.
$$

satisfies

$$
p(0)=0 .
$$

We solve problem (38) and (39), using a backward and an inward system with linear boundary feedbacks $\mathcal{I}_{U}$ : First given $f_{0}$ in $\mathcal{H}$, we consider $f \in C([0, T], \mathcal{H})$ the unique solution of

$$
\left\{\begin{array}{l}
\frac{\partial f}{\partial t}+A_{1} f-\mathcal{I}_{U} f=0 \text { in } \mathcal{H}, t \geq 0, \\
f(T)=f_{0} .
\end{array}\right.
$$

Applying Theorem 4.1 we get

$$
\mathcal{E}(f(t)) \leq M e^{-\omega(T-t)} \mathcal{E}\left(f_{0}\right) .
$$


Second we consider $g \in C([0, T], \mathcal{H})$ the unique solution of

$$
\left\{\begin{array}{l}
\frac{\partial g}{\partial t}+A_{1} g+\mathcal{I}_{U} g=0 \text { in } \mathcal{H}, t \geq 0, \\
g(0)=f(0) .
\end{array}\right.
$$

We now take $p=g-f$. From (40) and (42), $p$ satisfies (38) with

$$
K=-\mathcal{I}_{U} g-\mathcal{I}_{U} f .
$$

Let us further consider the mapping $\Lambda$ from $\mathcal{H}$ to $\mathcal{H}$ defined by

$$
\Lambda\left(f_{0}\right)=g(T) .
$$

We show that for $T>0$ such that $d:=M e^{-\omega T}<1$, the mapping $\Lambda-I$ is invertible by proving that $\|\Lambda\|_{\mathcal{L}(\mathcal{H}, \mathcal{H})}=\sqrt{d}$ using the estimate (41). Once $\Lambda-I$ is invertible, for any $p_{0} \in \mathcal{H}$, there exists a unique $f_{0} \in \mathcal{H}$ such that

$$
p_{0}=p(T)=g(T)-f(T)=(\Lambda-I) f_{0} .
$$

The proof will be complete if we can show that $K \in L^{2}(] 0, T[; U)$. For that purpose, we remark that the identity (32) yields

$$
\begin{aligned}
\mathcal{E}(f(T))-\mathcal{E}(f(0)) & =\int_{0}^{T}\left\|I_{U} f(t)\right\|_{U}^{2} d t, \\
\mathcal{E}(g(0))-\mathcal{E}(g(T)) & =\int_{0}^{T}\left\|I_{U} g(t)\right\|_{U}^{2} d t .
\end{aligned}
$$

Summing these two identities and using the initial condition of problem (42) and the final condition of (40), we obtain

$$
\int_{0}^{T}\left(\left\|I_{U} f(t)\right\|_{U}^{2}+\left\|I_{U} g(t)\right\|_{U}^{2}\right) d t=\mathcal{E}(f(T))-\mathcal{E}(g(T)) \leq \frac{1}{2}\left\|f_{0}\right\|_{\mathcal{H}}^{2} .
$$

Using the identity (44) and the boundedness of $(I-\Lambda)^{-1}$ we finally arrive at the estimate

$$
\int_{0}^{T}\left(\left\|I_{U} f(t)\right\|_{U}^{2}+\left\|I_{U} g(t)\right\|_{U}^{2}\right) d t \leq \frac{1}{2}\left\|(I-\Lambda)^{-1} p_{0}\right\|_{\mathcal{H}}^{2} \leq \frac{1}{2(1-\sqrt{d})^{2}}\left\|p_{0}\right\|_{\mathcal{H}}^{2} .
$$

This proves that $K$ given by (43) belongs to $L^{2}(] 0, T[; U)$.

Let us now pass to stability results in the nonlinear case. Using Liu's principle 15. and an integral inequality from 3 we deduce decay rates of the energy using appropriate nonlinear feedbacks. For that purpose we assume that the control space $U$ is of the form

$$
U=\prod_{j=1}^{J} U_{j},
$$

where for all $j=1, \cdots, J \in \mathbb{N}^{\star}:=\mathbb{N} \backslash\{0\}, U_{j}$ is a closed subspace of $L^{2}\left(X_{j}, \mu_{j}\right)^{N_{j}}$, when $\left(X_{j}, \mathcal{A}_{j}, \mu_{j}\right)$ is a measure space such that $\mu_{j}\left(X_{j}\right)<\infty$ and $N_{j} \in \mathbb{N}^{\star}$. For all $j=1, \cdots, J$, we suppose given a mapping $g_{j}: \mathbb{R}^{N_{j}} \rightarrow \mathbb{R}^{N_{j}}$ such that

$$
\begin{aligned}
& \left(g_{j}(x)-g_{j}(y)\right) \cdot(x-y) \geq 0, \forall x, y \in \mathbb{R}^{N_{j}} \text { (monotonicity), } \\
& g_{j}(0)=0 \\
& \left|g_{j}(x)\right| \leq M(1+|x|), \forall x \in \mathbb{R}^{N_{j}},
\end{aligned}
$$


for some positive constant $M$. We finally suppose that $B$ is given by

$$
\langle B u, v\rangle=\sum_{j=1}^{J} \int_{X_{j}} g_{j}\left(\left(I_{U} u\right)_{j}\left(x_{j}\right)\right) \cdot\left(I_{U} v\right)_{j}\left(x_{j}\right) d \mu_{j}\left(x_{j}\right),
$$

where we recall that $I_{U}$ is the embedding from $\mathcal{V}$ to $U$ and therefore $\left(I_{U} u\right)_{j}$ is the $j$ th component of $I_{U} u$.

Recall that the conditions (47) and (48) guarantee the assumption (29) on $B$, while (49) guarantees that $B$ is well defined. In most examples these conditions guarantee that the assumptions (24) and (25) hold. We further remark that these conditions always hold for $g_{j}(x)=x$, corresponding to linear controls, i.e., $B=\mathcal{I}_{U}$.

We now recall the integral inequality obtained in 3] (compare with Theorem 9.1 of [8]).

Theorem 4.3. Let $\mathcal{E}:[0,+\infty) \rightarrow[0,+\infty)$ be a nonincreasing mapping satisfying

$$
\int_{S}^{\infty} \phi(\mathcal{E}(t)) d t \leq T \mathcal{E}(S), \forall S \geq 0
$$

for some $T>0$ and some strictly increasing convex mapping $\phi$ from $[0,+\infty)$ to $[0,+\infty)$ such that $\phi(0)=0$. Then there exist $t_{1}>0$ and $c_{1}$ depending on $T$ and $\mathcal{E}(0)$ such that

$$
\mathcal{E}(t) \leq \phi^{-1}\left(\frac{\psi^{-1}\left(c_{1} t\right)}{c_{1} T t}\right), \forall t \geq t_{1}
$$

where $\psi$ is defined by

$$
\psi(t)=\int_{t}^{\infty} \frac{1}{\phi(s)} d s, \forall t>0 .
$$

We now give the consequence of this result to our system (30).

Theorem 4.4. Assume that the assumptions (24) to (29) hold for the pairs $\left(A_{1}, B\right)$ and $\left(A_{1}, \mathcal{I}_{U}\right)$. Let $g_{j}, j=1, \cdots, J$ satisfy (47) and (48) as well as

$$
\begin{aligned}
& g_{j}(x) \cdot x \geq m|x|^{2}, \forall x \in \mathbb{R}^{N_{j}}:|x| \geq 1, \\
& |x|^{2}+\left|g_{j}(x)\right|^{2} \leq G\left(g_{j}(x) \cdot x\right), \forall x \in \mathbb{R}^{N_{j}}:|x| \leq 1,
\end{aligned}
$$

for some positive constant $m$ and a concave strictly increasing function $G:[0, \infty) \rightarrow$ $[0, \infty)$ such that $G(0)=0$. If the pair $\left(-A_{1}, \mathcal{I}_{U}\right)$ satisfies the stability estimate, then there exist $c_{2}, c_{3}>0$ and $T_{1}>0$ (depending on $T, \mathcal{E}(0), \mu_{j}\left(X_{j}\right), j=1, \cdots, J$ ) such that

$$
\mathcal{E}(t) \leq c_{3} G\left(\frac{\psi^{-1}\left(c_{2} t\right)}{c_{2} T t}\right), \forall t \geq T_{1},
$$

for all solutions $u$ of (30), where $\psi$ is given by (53) for $\phi$ defined by

$$
\phi(s)=T \mu G^{-1}\left(\frac{s}{c_{3}}\right),
$$

where $\mu=\min _{j=1, \cdots, J} \mu_{j}\left(X_{j}\right)$.

Proof. By the density of $D(\mathcal{A})$ into $\mathcal{H}$, it suffices to prove (56) for data in $D(\mathcal{A})$. In that case let $u$ be the (strong) solution of (30) and consider $p$ the solution of problems 
(38) and (39) with $p_{0}=u(T) \in D(\mathcal{A})$ with $T>0$ sufficiently large (whose existence was established in Theorem 4.2).

By (30) and (38) we may write

$$
\left\langle\partial_{t} u+A_{1} u+B u, p\right\rangle_{\mathcal{V}^{\prime}, \mathcal{V}}+\left\langle\partial_{t} p+A_{1} p-K, u\right\rangle_{\mathcal{V}^{\prime}, \mathcal{V}}=0 .
$$

This may be written equivalently as

$$
\begin{aligned}
\left(\partial_{t} u, p\right)_{\mathcal{H}} & +\left(\partial_{t} p, u\right)_{\mathcal{H}}+\left\langle A_{1} u, p\right\rangle_{\mathcal{V}^{\prime}, \mathcal{V}}+\left\langle A_{1} p, u\right\rangle_{\mathcal{V}^{\prime}, \mathcal{V}} \\
& +\langle B u, p\rangle_{\mathcal{V}^{\prime}, \mathcal{V}}-\langle K, u\rangle_{\mathcal{V}^{\prime}, \mathcal{V}}=0 .
\end{aligned}
$$

As the assumption (28) yields

$$
\left\langle A_{1} u, p\right\rangle_{\mathcal{V}^{\prime}, \mathcal{V}}+\left\langle A_{1} p, u\right\rangle_{\mathcal{V}^{\prime}, \mathcal{V}}=0,
$$

the above identity reduces to

$$
\left(\partial_{t} u, p\right)_{\mathcal{H}}+\left(\partial_{t} p, u\right)_{\mathcal{H}}+\langle B u, p\rangle_{\mathcal{V}^{\prime}, \mathcal{V}}-\langle K, u\rangle_{\mathcal{V}^{\prime}, \mathcal{V}}=0
$$

Integrating this identity for $t \in(0, T)$, we get

$$
(u(T), p(T))_{\mathcal{H}}-(u(0), p(0))_{\mathcal{H}}+\int_{0}^{T}\left(\langle B u, p\rangle_{\mathcal{V}^{\prime}, \mathcal{V}}-\langle K, u\rangle_{\mathcal{V}^{\prime}, \mathcal{V}}\right) d t=0 .
$$

By the definitions of $K$ and $B$ we arrive at

$$
\begin{aligned}
(u(T), p(T))_{\mathcal{H}}-(u(0), p(0))_{\mathcal{H}} & =\int_{0}^{T}\left(\left(K, I_{U} u\right)_{U}\right. \\
& \left.-\sum_{j=1}^{J} \int_{X_{j}} g_{j}\left(\left(I_{U} u\right)_{j}\left(x_{j}\right)\right) \cdot\left(I_{U} p\right)_{j}\left(x_{j}\right) d \mu_{j}\left(x_{j}\right)\right) d t .
\end{aligned}
$$

Using the initial and final conditions on $p$, we have obtained

$$
2 \mathcal{E}(T)=\int_{0}^{T}\left(\left(K, I_{U} u\right)_{U}-\sum_{j=1}^{J} \int_{X_{j}} g_{j}\left(\left(I_{U} u\right)_{j}\left(x_{j}\right)\right) \cdot\left(I_{U} p\right)_{j}\left(x_{j}\right) d \mu_{j}\left(x_{j}\right)\right) d t
$$

Cauchy-Schwarz's inequality leads finally to

$$
\begin{aligned}
& 2 \mathcal{E}(T) \leq\|K\|_{L^{2}(0, T ; U)}\left\|I_{U} u\right\|_{L^{2}(0, T ; U)} \\
& +\left\|I_{U} p\right\|_{L^{2}(0, T ; U)}\left(\sum_{j=1}^{J} \int_{0}^{T} \int_{X_{j}}\left|g_{j}\left(\left(I_{U} u\right)_{j}\left(x_{j}\right)\right)\right|^{2} d \mu_{j}\left(x_{j}\right) d t\right)^{1 / 2} .
\end{aligned}
$$

Let us remark that the estimate (45) and the final conditions on $p$ yield

$$
\int_{0}^{T}\left(\left\|I_{U} f(t)\right\|_{U}^{2}+\left\|I_{U} g(t)\right\|_{U}^{2}\right) d t \leq \frac{1}{(1-\sqrt{d})^{2}} \mathcal{E}(T) .
$$

This estimate, the definition of $K$ and $p=g-f$, lead to

$$
\begin{aligned}
\int_{0}^{T}\|K(t)\|_{U}^{2} d t & \leq \frac{2}{(1-\sqrt{d})^{2}} \mathcal{E}(T), \\
\int_{0}^{T}\left\|I_{U} p(t)\right\|_{U}^{2} d t & \leq \frac{2}{(1-\sqrt{d})^{2}} \mathcal{E}(T) .
\end{aligned}
$$


Inserting these estimates into (58) we arrive at

$$
\mathcal{E}(T) \leq \frac{1}{(1-\sqrt{d})^{2}}\left(\sum_{j=1}^{J} \int_{0}^{T} \int_{X_{j}}\left\{\left|\left(I_{U} u\right)_{j}\left(x_{j}\right)\right|^{2}+\left|g_{j}\left(\left(I_{U} u\right)_{j}\left(x_{j}\right)\right)\right|^{2}\right\} d \mu_{j}\left(x_{j}\right) d t\right) .
$$

Using the properties on $g_{j}$ we can estimate this right-hand side and obtain

$$
\mathcal{E}(0) \leq c_{3} G\left(\frac{\mathcal{E}(0)-\mathcal{E}(T)}{T \mu}\right),
$$

for some $c_{3}>0$ (depending on $T, \mathcal{E}(0), \max _{j} \mu_{j}\left(X_{j}\right)$, and $\min _{j} \mu_{j}\left(X_{j}\right)$ ).

Using this argument in $[t, t+T]$ instead of $[0, T]$ we have shown that

$$
\mathcal{E}(t) \leq c_{3} G\left(\frac{\mathcal{E}(t)-\mathcal{E}(t+T)}{T \mu}\right)=\phi^{-1}(\mathcal{E}(t)-\mathcal{E}(t+T)), \forall t \geq 0,
$$

when we recall that $\phi$ was defined by (57).

As $\phi$ is increasing, this estimate implies that for all $0 \leq S<N$, with $N$ large enough

$$
\begin{aligned}
\int_{S}^{N} \phi(\mathcal{E}(t)) d t & \leq \int_{S}^{N}(\mathcal{E}(t)-\mathcal{E}(t+T)) d t \\
& \leq T \mathcal{E}(S)-T \mathcal{E}(N+T) .
\end{aligned}
$$

Letting $N$ goes to infinity we arrive at the estimate (51) (see Lemma 5.1 of [3]) and we conclude by Theorem 4.3 .

5. Proof of Theorem 2.2. Deriving (8) in time and integrating by parts in space we readily see that

$$
E^{\prime}(t)=-\frac{\alpha}{\beta} \int_{\Omega}|\nabla \theta(x, t)|^{2} d x-\int_{\Gamma_{2}} g\left(u^{\prime}(t)\right) \cdot u^{\prime}(t) d \Gamma-\int_{\Omega} f\left(u^{\prime}(t)\right) \cdot u^{\prime}(t) d x,
$$

and consequently

$$
\begin{aligned}
E(T)-E(S) & =-\frac{\alpha}{\beta} \int_{S}^{T} \int_{\Omega}|\nabla \theta|^{2} d x d t \\
& -\int_{S}^{T} \int_{\Gamma_{2}} g\left(u^{\prime}(t)\right) \cdot u^{\prime}(t) d \Gamma d t \\
& -\int_{S}^{T} \int_{\Omega} f\left(u^{\prime}(t)\right) \cdot u^{\prime}(t) d x d t, \forall 0 \leq S \leq T<\infty .
\end{aligned}
$$

The hypotheses (5), (6) and (7) lead to the decay of the energy.

Under additional hypotheses on $f$ and $g$, we will now obtain different types of decay. For that purpose introduce the constant

$$
R_{0}=\max _{x \in \bar{\Omega}}\left(\sum_{k=1}^{n}\left(x_{k}-x_{0 k}\right)^{2}\right)^{1 / 2} .
$$

Further, let $\gamma$ and $\lambda_{0}$ be the smallest positive constants such that for all $u \in\left(H_{\Gamma_{1}}^{1}(\Omega)\right)^{n}$

$$
\int_{\Gamma_{2}}|u|^{2} d \Gamma \leq \gamma^{2}\left(\int_{\Omega} \sigma(u): \varepsilon(u) d x+\int_{\Gamma_{2}} a|u|^{2} d \Gamma\right)
$$


and

$$
\|u\|_{\left(L^{2}(\Omega)\right)^{n}}^{2} \leq \lambda_{0}^{2}\left(\int_{\Omega} \sigma(u): \varepsilon(u) d x+\int_{\Gamma_{2}} a|u|^{2} d \Gamma\right)
$$

respectively.

To prove Theorem 2.2, we are reduced to check the sufficient conditions of Theorem 4.4. In our case it remains to show that the linear system associated with (3) is exponentially stable. This system takes the form:

$$
\left\{\begin{array}{lcc}
u^{\prime \prime}-\operatorname{div} \sigma(u)+\alpha \nabla \theta+u^{\prime} & =0 \text { in } Q, \\
\theta^{\prime}-\Delta \theta+\beta \operatorname{div} u^{\prime} & =0 \text { in } Q, \\
\theta & =0 \text { on } \Sigma, \\
u & =0 \text { on } \Sigma_{1}, \\
\sigma(u) \cdot \nu+a u+u^{\prime} & =0 \text { on } \Sigma_{2}, \\
u(., 0)=u_{0}, u^{\prime}(., 0)=u_{1}, \theta(., 0)=\theta_{0} & & \text { in } \Omega .
\end{array}\right.
$$

Recall that this system is dissipative and that its energy defined by (8) satisfies

$$
\begin{aligned}
E(T)-E(S) & =-\frac{\alpha}{\beta} \int_{S}^{T} \int_{\Omega}|\nabla \theta|^{2} d x d t \\
& -\int_{S}^{T} \int_{\Gamma_{2}}\left|u^{\prime}(t)\right|^{2} d \Gamma d t \\
& -\int_{S}^{T} \int_{\Omega}\left|u^{\prime}(t)\right|^{2} d x d t \leq 0, \forall 0 \leq S \leq T<\infty .
\end{aligned}
$$

To enter in the formalism of the previous section, we have to define the operators $A_{1}$ and $\mathcal{I}_{U}$ associated with our system (62): $A_{1}$ is defined on $\mathcal{V}:=\left(H_{\Gamma_{1}}^{1}\right)^{n} \times\left(H_{\Gamma_{1}}^{1}\right)^{n} \times$ $H_{0}^{1}(\Omega)$ by

$$
A_{1} \Phi=(-v, A u+\alpha \nabla \theta, \beta \operatorname{div} v) .
$$

Moreover, in view of the feedbacks used in (62) and of the identities (32) and (63), we need to take

$$
U=\left(L^{2}\left(\Gamma_{2}\right)\right)^{n} \times\left(L^{2}(\Omega)\right)^{n} \times\left(L^{2}(\Omega)\right)^{n}
$$

and to define the mappings

$$
\begin{gathered}
I_{U}: \mathcal{V} \longmapsto U \\
(u, v, \theta) \longmapsto\left(v_{\mid \Gamma_{2}}, v, \nabla \theta\right)
\end{gathered}
$$

and $\mathcal{I}_{U}$ from $\mathcal{V}$ into $\mathcal{V}^{\prime}$ by

$$
\begin{aligned}
& \left\langle\mathcal{I}_{U}(u, v, \theta),\left(u^{*}, v^{*}, \theta^{*}\right)\right\rangle=\left(I_{U}(u, v, \theta), I_{U}\left(u^{*}, v^{*}, \theta^{*}\right)\right) \\
= & \int_{\Gamma_{2}} v \cdot v^{*} d \Gamma+\int_{\Omega} v \cdot v^{*} d x+\frac{\alpha}{\beta} \int_{\Omega} \nabla \theta \cdot \nabla \theta^{*} d x .
\end{aligned}
$$

To establish the exponential stability of the system (62), we are reduced to prove a stability estimate for the pair $\left(-A_{1}, \mathcal{I}_{U}\right)$ (see Theorem 4.1).

We start with two technical lemmas: 
Lemma 5.1. Let $(u, \theta)$ be a strong solution of (62). Define

$$
M(u)=2(m \cdot \nabla) u+(n-1) u .
$$

Then for all $t \geq 0$, there exists a positive constant $\eta$ such that

$$
\|M(u)\|_{\left(L^{2}(\Omega)\right)^{n}}^{2} \leq \eta E(t) .
$$

Proof. By integration by parts we have

$$
\begin{aligned}
\|M(u)\|_{\left(L^{2}(\Omega)\right)^{n}}^{2} & =\int_{\Omega}\left[|2(m \cdot \nabla) u|^{2}+(n-1)^{2}|u|^{2}+4(n-1) u \cdot(m \cdot \nabla) u\right] d x \\
& =\int_{\Omega}\left[|2(m \cdot \nabla) u|^{2}+(n-1)^{2}|u|^{2}+2(n-1) m \cdot \nabla\left(|u|^{2}\right)\right] d x \\
& =\int_{\Omega}\left[|2(m \cdot \nabla) u|^{2}+\left(1-n^{2}\right)|u|^{2}\right] d x+2(n-1) \int_{\Gamma_{2}} m \cdot \nu|u|^{2} d \Gamma \\
& \leq 4 R_{0}^{2} \int_{\Omega}|\nabla u|^{2} d x+2(n-1) \int_{\Gamma_{2}} m \cdot \nu|u|^{2} d \Gamma .
\end{aligned}
$$

We conclude using Korn's inequality.

Lemma 5.2. There exists a constant $C>0$ such that for all $\varepsilon \in(0,1)$ and $T>0$, we have

$$
\int_{0}^{T} \int_{\Gamma_{2}} a|u|^{2} d \Gamma d t \leq \frac{C}{\varepsilon} E(0)+\varepsilon \int_{0}^{T} E(t) d t .
$$

Proof. We proceed as in [1]. For $t \geq 0$, consider the solution $z=z(t)$ of

$$
\left\{\begin{array}{l}
\operatorname{div}(\sigma(z))=0 \text { in } \Omega, \\
z=u \text { on } \Gamma .
\end{array}\right.
$$

This solution is characterized by $z=\omega+u$ where $\omega \in\left(H_{0}^{1}(\Omega)\right)^{n}$ is the unique solution of

$$
\int_{\Omega} \sigma(\omega): \varepsilon(v) d x=-\int_{\Omega} \sigma(u): \varepsilon(v) d x, \quad \forall v \in\left(H_{0}^{1}(\Omega)\right)^{n} .
$$

This identity means that

$$
\int_{\Omega} \sigma(z): \varepsilon(v) d x=0, \quad \forall v \in\left(H_{0}^{1}(\Omega)\right)^{n} .
$$

Taking $v=z-u$ in this identity, we deduce that

$$
\int_{\Omega} \sigma(z): \varepsilon(u) d x=\int_{\Omega} \sigma(z): \varepsilon(z) d x \geq 0 .
$$

Let us now show that $z$ also satisfies

$$
\int_{\Omega} f \cdot z d x=-\int_{\Gamma} z \cdot\left(\sigma\left(v_{f}\right) \nu\right) d \Gamma, \quad \forall f \in\left(L^{2}(\Omega)\right)^{n},
$$

where $v_{f} \in\left(H_{0}^{1}(\Omega)\right)^{n}$ is the unique solution of

$$
\int_{\Omega} \sigma\left(v_{f}\right): \varepsilon(w) d x=\int_{\Omega} f \cdot w d x, \forall w \in\left(H_{0}^{1}(\Omega)\right)^{n} .
$$


Indeed from (65) and the property $\sigma(z): \epsilon(v)=\sigma(v): \epsilon(z)$ (consequence of the assumption $a_{i j k l}=a_{k l i j}$ ), we may write

$$
\int_{\Omega} \sigma\left(v_{f}\right): \varepsilon(z) d x=0
$$

By Green's formula, we deduce that

$$
-\int_{\Omega} \operatorname{div} \sigma\left(v_{f}\right) \cdot z d x+\int_{\Gamma} z \cdot\left(\sigma\left(v_{f}\right) \nu\right) d \Gamma=0 .
$$

This proves the identity (67) since $\operatorname{div} \sigma\left(v_{f}\right)=-f$.

In the identity (67) taking $f=z$, we may write

$$
\int_{\Omega}|z|^{2} d x=-\int_{\Gamma} z \cdot\left(\sigma\left(v_{z}\right) \nu\right) d \Gamma .
$$

Since $z=u$ on $\Gamma$ and $u=0$ on $\Gamma_{1}$, by Cauchy-Schwarz's inequality we obtain

$$
\int_{\Omega}|z|^{2} d x \leq\|u\|_{\left(L^{2}\left(\Gamma_{2}\right)\right)^{n}}\left\|\sigma\left(v_{z}\right) \nu\right\|_{\left(L^{2}(\Gamma)\right)^{n}}
$$

As the boundary $\Gamma$ is $C^{2}$, elliptic regularity results yield $v_{z} \in\left(H^{2}(\Omega)\right)^{n}$ with the estimate

$$
\left\|v_{z}\right\|_{\left(H^{2}(\Omega)\right)^{n}} \leq K\|z\|_{\left(L^{2}(\Omega)\right)^{n}},
$$

for some positive constant $K$. This estimate and a standard trace theorem lead to

$$
\left\|\sigma\left(v_{z}\right) \nu\right\|_{\left(L^{2}(\Gamma)\right)^{n}} \leq K_{1}\|z\|_{\left(L^{2}(\Omega)\right)^{n}},
$$

for some positive constant $K_{1}$. Inserting this estimate in (68) we arrive at

$$
\int_{\Omega}|z|^{2} d x \leq C_{0} \int_{\Gamma_{2}}|u|^{2} d \Gamma,
$$

where $C_{0}$ is a positive constant.

Since $z^{\prime}$ is a solution of problem (64) with $u^{\prime}$ instead of $u$, the above arguments yield

$$
\int_{\Omega}\left|z^{\prime}\right|^{2} d x \leq C_{0} \int_{\Gamma_{2}}\left|u^{\prime}\right|^{2} d \Gamma
$$

For $0<T<\infty$, we set

$$
\begin{aligned}
Q_{T} & =\Omega \times[0, T], \\
\Sigma_{T} & =\Gamma \times[0, T] ; \Sigma_{1 T}=\Gamma_{1} \times[0, T] ; \quad \Sigma_{2 T}=\Sigma_{T} \backslash \Sigma_{1 T} .
\end{aligned}
$$

Multiplying the first identity of (62) by $z$ and integrating on $Q_{T}$, we obtain

$$
\int_{Q_{T}} z\left(u^{\prime \prime}-\operatorname{div}(\sigma(u))+\alpha \nabla \theta+u^{\prime}\right) d x d t=0 .
$$

Applying Green's formula and taking into account the boundary conditions in (62) and in (64), we get

$$
\begin{gathered}
\int_{Q_{T}}\left(z u^{\prime \prime}+\sigma(z): \varepsilon(u)+\alpha z \nabla \theta+u^{\prime} z\right) d x d t \\
+\int_{\Sigma_{2 T}} u\left(a u+u^{\prime}\right) d \Sigma=0 .
\end{gathered}
$$


Integrating by parts in $t$ and using (66), we obtain (using again the property $\sigma(z)$ : $\epsilon(u)=\sigma(u): \epsilon(z))$

$$
\begin{aligned}
\int_{\Sigma_{2 T}} a|u|^{2} d \Sigma & \leq-\int_{\Sigma_{2 T}} u u^{\prime} d \Sigma+\int_{Q_{T}} z^{\prime} u^{\prime} d x d t \\
& -\alpha \int_{Q_{T}} z \nabla \theta d x d t-\int_{Q_{T}} u^{\prime} z d x d t-\left.\int_{\Omega} z u^{\prime}\right|_{0} ^{T} .
\end{aligned}
$$

Fix an arbitrary $\varepsilon_{0}>0$. Using several times (63), (69), (70) and Young's inequality, we can estimate the different integrals of the right-hand side of the above inequality as follows:

$$
\begin{aligned}
-\int_{\Sigma_{2 T}} u u^{\prime} d \Sigma & \leq \varepsilon_{0} \int_{\Sigma_{2 T}}|u|^{2} d \Sigma+\frac{1}{4 \varepsilon_{0}} \int_{\Sigma_{2 T}}\left|u^{\prime}\right|^{2} d \Sigma \\
& \leq 2 \varepsilon_{0} \gamma^{2} \int_{0}^{T} E(t) d t+\frac{1}{4 \varepsilon_{0}} E(0), \\
-\int_{Q_{T}} z^{\prime} u^{\prime} d x d t & \leq \varepsilon_{0} \int_{Q_{T}}\left|u^{\prime}\right|^{2} d x d t+\frac{1}{4 \varepsilon_{0}} \int_{Q_{T}}\left|z^{\prime}\right|^{2} d x d t \\
& \leq 2 \varepsilon_{0} \int_{0}^{T} E(t) d t+\frac{C_{0}}{4 \varepsilon_{0}} E(0), \\
-\int_{Q_{T}} \alpha \nabla \theta \cdot z d x d t & \leq \frac{\alpha^{2}}{4 \varepsilon_{0}} \int_{Q_{T}}|\nabla \theta|^{2} d x d t+\varepsilon_{0} \int_{Q_{T}}|z|^{2} d \Sigma \\
& \leq \frac{\alpha \beta}{4 \varepsilon_{0}} E(0)+2 \varepsilon_{0} C_{0} \gamma^{2} \int_{0}^{T} E(t) d t \\
\int_{Q_{T}} u^{\prime} z d x d t & \leq \varepsilon_{0} \int_{Q_{T}}|z|^{2} d x d t+\frac{1}{4 \varepsilon_{0}} \int_{Q_{T}}\left|u^{\prime}\right|^{2} d \Sigma \\
\leq & \varepsilon_{0} C_{0} \gamma^{2} \int_{0}^{T} E(t) d t+\frac{1}{4 \varepsilon_{0}} E(0), \\
\left.\int_{\Omega} z u^{\prime}\right|_{0} ^{T} & \leq 4\left(1+C_{0} \gamma^{2}\right) E(0) .
\end{aligned}
$$

Using these different estimates, we arrive at the requested estimate by taking $\varepsilon_{0}=$ $\frac{\varepsilon}{2 \gamma^{2}+2+3 C_{0} \gamma^{2}}$ and $C=\left(\frac{1}{2}+\frac{C_{0}}{4}+\frac{\alpha \beta}{4}+4\left(1+C_{0} \gamma^{2}\right)\right)\left(2 \gamma^{2}+2+3 C_{0} \gamma^{2}\right)$.

Proof of Theorem 2.2. Multiplying the first identity of (62) by

$$
M(u)=2(m \cdot \nabla) u+(n-1) u
$$

and integrating by parts on $Q_{T}$ we obtain

$$
\int_{Q_{T}} u^{\prime \prime} \cdot M(u) d x d t=\left.\left(u^{\prime}, M(u)\right)\right|_{0} ^{T}-\int_{\Sigma_{2 T}} m \cdot \nu\left|u^{\prime}\right|^{2} d \Sigma+\int_{Q_{T}}\left|u^{\prime}\right|^{2} d x d t
$$




$$
\begin{aligned}
\int_{Q_{T}} \operatorname{div} \sigma(u) \cdot M(u) d x d t & =\int_{\Sigma_{T}}[(\sigma(u) \cdot \nu) \cdot M(u)-(m \cdot \nu) \sigma(u): \varepsilon(u)] d \Sigma \\
& -\int_{Q_{T}} \sigma(u): \varepsilon(u) d x d t \\
& +\int_{Q_{T}} m_{p} \partial_{p}\left(a_{i j k l}\right) \varepsilon_{i j}(u) \varepsilon_{k l}(u) d x d t .
\end{aligned}
$$

The two above identities combined with the boundary conditions in (62) (implying in particular $\frac{\partial u_{i}}{\partial x_{k}}=\frac{\partial u_{i}}{\partial \nu} \nu_{k}$ on $\Sigma_{1 T}$ ) lead to

$$
\begin{aligned}
& 2 \int_{0}^{T} E(t) d t=-\left.\left(u^{\prime}, M(u)\right)\right|_{0} ^{T}+\int_{\Sigma_{2 T}}\left(m \cdot \nu\left|u^{\prime}\right|^{2}+a|u|^{2}\right) d \Sigma-\int_{Q_{T}} u^{\prime} M(u) d x d t \\
- & \alpha \int_{Q_{T}} \nabla \theta \cdot M(u) d x d t+\frac{\alpha}{\beta} \int_{Q_{T}} \theta^{2} d x d t \\
+ & \int_{\Sigma_{T}}[(\sigma(u) \nu) M(u)-m \cdot \nu \sigma(u): \varepsilon(u)] d \Sigma \\
+ & \int_{Q_{T}} m_{p} \partial_{p}\left(a_{i j k l}\right) \varepsilon_{i j}(u) \varepsilon_{k l}(u) d x d t .
\end{aligned}
$$

This identity may be equivalently written

$$
2 \int_{0}^{T} E(t) d t=\sum_{i=1}^{6} I_{i}
$$

where we have set

$$
\begin{aligned}
I_{1} & :=-\left.\left(u^{\prime}, M(u)\right)\right|_{0} ^{T}, \\
I_{2} & :=\int_{\Sigma_{2 T}}\left(m \cdot \nu\left|u^{\prime}\right|^{2}+a|u|^{2}\right) d \Sigma, \\
I_{3} & :=-\int_{Q_{T}} u^{\prime} \cdot M(u) d x d t, \\
I_{4} & :=\alpha \int_{Q_{T}}\left[\nabla \theta \cdot M(u) d x d t+\frac{\alpha}{\beta} \theta^{2}\right] d x d t, \\
I_{5} & :=\int_{\Sigma_{T}}[(\sigma(u) \nu) \cdot M(u)-m \cdot \nu \sigma(u): \varepsilon(u)] d \Sigma, \\
I_{6} & :=\int_{Q_{T}} m_{p} \partial_{p}\left(a_{i j k l}\right) \varepsilon_{i j}(u) \varepsilon_{k l}(u) d x d t .
\end{aligned}
$$

It then remains to estimate each term $I_{i}$ : For $t \geq 0$ and $\varepsilon>0$ fixed, we have

$$
\int_{\Omega} u^{\prime}(t) \cdot M(u)(t) d x \leq \frac{1}{2 \varepsilon}\left\|u^{\prime}(t)\right\|_{\left(L^{2}(\Omega)\right)^{n}}^{2}+\frac{\varepsilon}{2}\|M(u(t))\|_{\left(L^{2}(\Omega)\right)^{n}}^{2}
$$

By Lemma 5.1 and the identity (63) , there exist two positive constants $k_{1}$ and $k_{3}$ such that

$$
I_{1} \leq k_{1} E(0)
$$


and

$$
I_{3} \leq \frac{k_{3}}{\varepsilon} \int_{Q_{T}}\left|u^{\prime}\right|^{2} d x d t+k_{3} \varepsilon \int_{0}^{T} E(t) d t .
$$

Similarly, by Lemma 5.2, there exists a positive constant $k_{2}$ such that

$$
I_{2} \leq R_{0} \int_{\Sigma_{2 T}}\left|u^{\prime}\right|^{2} d \Sigma+\int_{\Sigma_{2 T}} a|u|^{2} d \Sigma \leq R_{0} \int_{\Sigma_{2 T}}\left|u^{\prime}\right|^{2} d \Sigma+\frac{k_{2}}{\varepsilon} E(0)+\varepsilon \int_{0}^{T} E(t) d t .
$$

By Young's inequality, the definition of $R_{0}$ and of $\lambda_{0}$, and taking into account (59), we have successively

$$
\begin{aligned}
I_{4} & \leq \frac{\alpha^{2}(n-1)^{2}}{4 \varepsilon} \int_{Q_{T}}|\nabla \theta|^{2}+\varepsilon \int_{Q_{T}}|u|^{2}+\frac{\alpha^{2} R_{0}^{2}}{\varepsilon} \int_{Q_{T}}|\nabla \theta|^{2}+\varepsilon \int_{Q_{T}}|\nabla u|^{2} \\
& +\frac{\alpha}{\beta} \int_{Q_{T}}|\theta|^{2} d x d t, \\
& \leq\left[\frac{\alpha \beta(n-1)^{2}}{4 \varepsilon}+\frac{\alpha \beta R_{0}^{2}}{\varepsilon}+\lambda_{0}^{2}\right] \int_{Q_{T}} \frac{\alpha}{\beta}|\nabla \theta|^{2}+\varepsilon \int_{Q_{T}}\left[|u|^{2}+|\nabla u|^{2}\right] d x d t \\
& \leq\left[\frac{\alpha \beta(n-1)^{2}}{4 \varepsilon}+\frac{\alpha \beta R_{0}^{2}}{\varepsilon}+\lambda_{0}^{2}\right] \int_{Q_{T}} \frac{\alpha}{\beta}|\nabla \theta|^{2}+\varepsilon k_{4} \int_{0}^{T} E(t) d t \\
& \leq k_{4}^{\prime} E(0)+\varepsilon k_{4} \int_{0}^{T} E(t) d t,
\end{aligned}
$$

where $k_{4}$ and $k_{4}^{\prime}$ are positive constants.

As in [1] the estimation of $I_{5}$ is based on the use of local coordinates systems. Namely for all $x \in \Gamma$, we denote by $\pi(x)$ the orthogonal projection on the tangent hyperplane $T_{x}(\Gamma)$. Any vector field $v: \bar{\Omega} \rightarrow \mathbb{R}^{n}$ will be split up as follows:

$$
v(x)=v_{T}(x)+v_{\nu}(x) \nu(x),
$$

where $v_{T}(x)=\pi(x) v(x)$ is the tangential component of $v$ and $v_{\nu}(x)=v(x) \cdot \nu(x)$. We further denote by $\partial_{\nu} v=\nu \cdot \nabla v$, the normal derivative of $v$ and by $\nabla_{T} v=\nabla v-\partial_{n} v$ the tangential gradient of $v$. For further use, we set $\partial_{T} v=\overline{\nabla_{T} v}$, the tangential derivation of $v$, where $\bar{\tau}$ means the transposed matrix of the matrix $\tau$. Similarly, for a vector $v, \bar{v}$ will mean its transposed vector.

Following 9 or 22 , the strain tensor is written as follows:

$$
\varepsilon(v)=\varepsilon_{T}(v)+\nu \overline{\varepsilon_{S}(v)}+\varepsilon_{S}(v) \bar{\nu}+\varepsilon_{\nu}(v) \nu \bar{\nu} \quad \text { on } \quad \Gamma,
$$

with

$$
\begin{aligned}
2 \varepsilon_{T}(v) & =\pi\left(\partial_{T} v_{T}\right) \pi+\pi \overline{\partial_{T} v_{T}} \pi+2 v_{\nu} \partial_{T} \nu \\
2 \varepsilon_{S}(v) & =\partial_{\nu} v_{T}+\nabla_{T} v_{\nu}-\left(\partial_{T} \nu\right) v_{T}, \\
\varepsilon_{\nu}(v) & =\partial_{\nu} v_{\nu}
\end{aligned}
$$

where $\left(\partial_{T} \nu\right)$ is the curvature operator of $\Gamma$. Similarly, the stress tensor may be written

$$
\sigma(v)=\sigma_{T}(v)+\nu \overline{\sigma_{S}(v)}+\sigma_{S}(v) \bar{\nu}+\sigma_{\nu}(v) \nu \bar{\nu} \quad \text { on } \quad \Gamma,
$$

where $\sigma_{T}(v)$ is an endomorphism on the tangent hyperplane, $\sigma_{S}(v)$ is a tangent vector field and $\sigma_{\nu}(v)$ is a scalar field. 
These splittings allow us to write

$$
\begin{array}{r}
\varepsilon(v): \varepsilon(v)=\varepsilon_{T}(v): \varepsilon_{T}(v)+2\left|\varepsilon_{S}(v)\right|^{2}+\left|\varepsilon_{\nu}(v)\right|^{2} \quad \text { on } \quad \Gamma, \\
\sigma(v): \varepsilon(v)=\sigma_{T}(v): \varepsilon_{T}(v)+2 \overline{\sigma_{S}(v)} \varepsilon_{S}(v)+\sigma_{\nu}(v) \varepsilon_{\nu}(v) \quad \text { on } \quad \Gamma .
\end{array}
$$

Let us now recall that Proposition 1 of [1] showed that there exists $C>0$ such that

$$
\int_{\Gamma}\left|\nabla_{T} v_{T}\right|^{2} d \Gamma \leq C \int_{\Gamma}\left(\left|v_{T}\right|^{2}+\varepsilon_{T}\left(v_{T}\right): \varepsilon_{T}\left(v_{T}\right)\right) d \Gamma, \forall v_{T} \in H^{1}(\Gamma)^{n-1} .
$$

The Dirichlet boundary conditions on $\Gamma_{1}$ lead to

$$
M(u)=2(m \cdot \nabla) u=2 m \cdot \nu \partial_{\nu} u, \quad \varepsilon_{T}(u)=0, \quad 2 \varepsilon_{S}(u)=\partial_{\nu} u_{T}
$$

and consequently,

$$
\begin{aligned}
(\sigma(u) \nu) \cdot M(u) & =2 m \cdot \nu\left(\overline{\sigma_{S}(u)} \partial_{\nu} u_{T}+\sigma_{\nu}(u) \partial_{\nu} u_{\nu}\right) \quad \text { on } \Gamma_{1} \\
\sigma(u): \varepsilon(u) & =\overline{\sigma_{S}(u)} \partial_{\nu} u_{T}+\sigma_{\nu}(u) \partial_{\nu} u_{\nu} \quad \text { on } \Gamma_{1} .
\end{aligned}
$$

These two identities lead to

$$
\int_{\Gamma_{1}}((\sigma(u) \nu) \cdot M(u)-m \cdot \nu \sigma(u): \varepsilon(u)) d \Gamma=\int_{\Gamma_{1}} m \cdot \nu \sigma(u): \varepsilon(u) d \Gamma .
$$

As $m \cdot \nu \leq 0$ on $\Gamma_{1}$ and $\sigma(u): \varepsilon(u) \geq 0$, we deduce that

$$
\int_{\Gamma_{1}}((\sigma(u) \nu) M(u)-m \cdot \nu \sigma(u): \varepsilon(u)) d \Gamma \leq 0 .
$$

Using the boundary conditions on $\Gamma_{2}$, we may write

$$
\int_{\Gamma_{2}}(\sigma(u) \nu) \cdot M(u) d \Gamma=-\int_{\Gamma_{2}} 2\left(a u+u^{\prime}\right) \cdot(m \cdot \nabla) u d \Gamma-(n-1) \int_{\Gamma_{2}} u \cdot\left(a u+u^{\prime}\right) d \Gamma .
$$

This identity, the estimate (72) and the assumption (41) lead to

$$
\begin{aligned}
I_{5} & \leq C \int_{\Sigma_{2 T}}\left(|u|^{2}+\left|u^{\prime}\right|^{2}\right) d \Sigma \\
& -\int_{0}^{T} \int_{\Gamma_{2}} 2\left(a u+u^{\prime}\right) \cdot(m \cdot \nabla) u d \Gamma d t \\
& -k \delta \int_{0}^{T} \int_{\Gamma_{2}}\left(\varepsilon_{T}(u): \varepsilon_{T}(u)+2\left|\varepsilon_{S}(u)\right|^{2}+\left|\varepsilon_{\nu}(u)\right|^{2}\right) d \Gamma d t,
\end{aligned}
$$

where here and below $C$ will denote a positive constant large enough but independent of $u$. $k$ is a positive constant such that $m \cdot \nu \geq k>0$ on $\Gamma_{2}$.

We first estimate the term

$$
\mathcal{I}_{1}=\int_{S}^{T} \int_{\Gamma_{2}} 2 a u \cdot(m \cdot \nabla) u d \Gamma d t
$$

We remark that $u \cdot(m \cdot \nabla) u=\frac{1}{2} m \cdot \nabla\left(|u|^{2}\right)$ and by writting $\nabla u=\nabla_{T} u+\nu\left(\partial_{\nu} u\right)$, we obtain

$$
u \cdot(m \cdot \nabla) u=\frac{1}{2} \nabla_{T}\left(|u|^{2}\right) \cdot m_{T}+m_{\nu} u_{T} \cdot\left(\partial_{\nu} u_{T}\right)+m_{\nu} u_{\nu}\left(\partial_{\nu} u_{\nu}\right) \quad \text { on } \quad \Gamma_{2} .
$$


By Green's formula, we have

and therefore

$$
\int_{\Gamma_{2}} a \nabla_{T}\left(|u|^{2}\right) \cdot m_{T} d \Gamma=-\int_{\Gamma_{2}}|u|^{2} \operatorname{div}_{T}\left(a m_{T}\right) d \Gamma
$$

$$
\left|\int_{\Gamma_{2}} a \nabla_{T}\left(|u|^{2}\right) \cdot m_{T} d \Gamma\right| \leq C \int_{\Gamma_{2}}|u|^{2} d \Gamma
$$

Using the expression of $\varepsilon_{S}(u)$, we may write

$$
m_{\nu} u_{T} \cdot \partial_{\nu} u_{T}=m_{\nu} u_{T} \cdot\left(2 \varepsilon_{S}(u)+\left(\partial_{T} \nu\right) u_{T}-\nabla_{T} u_{\nu}\right) \quad \text { on } \quad \Gamma_{2} .
$$

By Young's inequality we have

$$
\left|\int_{\Gamma_{2}} 4 a m_{\nu} u_{T} \cdot \varepsilon_{S}(u) d \Gamma\right| \leq \theta \int_{\Gamma_{2}}\left|\varepsilon_{S}(u)\right|^{2} d \Gamma+\frac{C}{\theta} \int_{\Gamma_{2}}\left|u_{T}\right|^{2} d \Gamma
$$

for any $\theta \in(0,1)$ (that will be chosen later on).

Green's formula and Leibniz's rule yield

$$
\begin{aligned}
\int_{\Gamma_{2}} a m_{\nu} u_{T} \cdot \nabla_{T} u_{\nu} d \Gamma & =-\int_{\Gamma_{2}} u_{\nu} \operatorname{div}_{T}\left(a m_{\nu} u_{T}\right) d \Gamma \\
& =-\int_{\Gamma_{2}} a m_{\nu} u_{\nu} \operatorname{div}_{T} u_{T} d \Gamma-\int_{\Gamma_{2}} u_{\nu} \nabla_{T}\left(a m_{\nu}\right) \cdot u_{T} d \Gamma .
\end{aligned}
$$

By the estimate (71) and again Young's inequality, we obtain

$$
\left|\int_{\Gamma_{2}} 2 a m_{\nu} u_{T} \cdot \nabla_{T} u_{\nu} d \Gamma\right| \leq \theta \int_{\Gamma_{2}} \varepsilon_{T}\left(u_{T}\right): \varepsilon_{T}\left(u_{T}\right) d \Gamma+\frac{C}{\theta} \int_{\Gamma_{2}}|u|^{2} d \Gamma .
$$

The remaining terms are estimated by Young's inequality to obtain

$$
\begin{aligned}
\left|\mathcal{I}_{1}\right| & \leq \theta \int_{0}^{T} \int_{\Gamma_{2}}\left(\left|\partial_{\nu} u_{\nu}\right|^{2}+\varepsilon_{T}\left(u_{T}\right): \varepsilon_{T}\left(u_{T}\right)+\left|\varepsilon_{S}(u)\right|^{2}\right) d \Gamma d t \\
& +\frac{C}{\theta} \int_{0}^{T} \int_{\Gamma_{2}}|u|^{2} d \Gamma d t .
\end{aligned}
$$

Next we estimate the term

$$
\mathcal{I}_{2}=\int_{0}^{T} \int_{\Gamma_{2}} 2 u^{\prime} \cdot(m \cdot \nabla) u d \Gamma d t
$$

The expression of the gradient in local coordinates gives

$$
\begin{aligned}
u^{\prime} \cdot(m \cdot \nabla) u & =u_{T}^{\prime} \cdot\left(\partial_{T} u_{T}\right) m_{T}+\left(u_{\nu} u_{T}^{\prime}-u_{\nu}^{\prime} u_{T}\right) \cdot\left(\partial_{T} \nu\right) m_{T} \\
& +u_{\nu}^{\prime} m_{T} \cdot \nabla_{T} u_{\nu}+m_{\nu}\left(u_{T}^{\prime} \cdot \partial_{\nu} u_{T}+u_{\nu}^{\prime}\left(\partial_{\nu} u_{\nu}\right)\right) \text { on } \Gamma_{2} .
\end{aligned}
$$

The most difficult terms are $u_{\nu}^{\prime} m_{T} \cdot \nabla_{T} u_{\nu}$ and $m_{\nu} u_{T}^{\prime} \cdot \partial_{\nu} u_{T}$, the other terms being estimated with the help of Young's inequality. For the first one, using local charts, a partition of unity and a Fourier analysis (see [1] for the details), one can show that

$$
\left|\int_{0}^{T} \int_{\Gamma_{2}} u_{\nu}^{\prime} m_{T} \cdot \nabla_{T} u_{\nu} d \Gamma d t\right| \leq C\left[\left\|u_{\nu}\right\|_{H^{1 / 2}\left(\Gamma_{2}\right)}^{2}\right]_{0}^{T} .
$$

By the definition of the energy, we then obtain

$$
\left|\int_{0}^{T} \int_{\Gamma_{2}} u_{\nu}^{\prime} m_{T} \cdot \nabla_{T} u_{\nu} d \Gamma d t\right| \leq C E(0)
$$


For the second term, we use the expression of $\varepsilon_{S}(u)$ to write

$$
m_{\nu} u_{T}^{\prime} \cdot \partial_{\nu} u_{T}=m_{\nu} u_{T}^{\prime} \cdot\left(2 \varepsilon_{S}(u)+\left(\partial_{T} \nu\right) u_{T}-\nabla_{T} u_{\nu}\right) \quad \text { on } \quad \Gamma_{2} .
$$

The two first terms are estimated by Young's inequality, for the last one, we perform integrations by parts to get

$$
\begin{aligned}
\int_{0}^{T} \int_{\Gamma_{2}} m_{\nu} u_{T}^{\prime} \cdot \nabla_{T} u_{\nu} d \Gamma d t & =\left[\int_{\Gamma_{2}} m_{\nu} u_{T} \cdot \nabla_{T} u_{\nu} d \Gamma\right]_{0}^{T} \\
& +\int_{0}^{T} \int_{\Gamma_{2}} \operatorname{div}_{T}\left(m_{\nu} u_{T}\right) u_{\nu}^{\prime} d \Gamma d t .
\end{aligned}
$$

For this last term by Leibniz's rule and the estimate (71), we have

$$
\begin{aligned}
\left|\int_{0}^{T} \int_{\Gamma_{2}} \operatorname{div}_{T}\left(m_{\nu} u_{T}\right) u_{\nu}^{\prime} d \Gamma d t\right| & \leq \theta \int_{0}^{T} \int_{\Gamma_{2}} \varepsilon_{T}\left(u_{T}\right): \varepsilon_{T}\left(u_{T}\right) d \Gamma d t \\
& +\frac{C}{\theta} \int_{0}^{T} \int_{\Gamma_{2}}\left(|u|^{2}+\left|u^{\prime}\right|^{2}\right) d \Gamma d t
\end{aligned}
$$

It remains to estimate $\int_{\Gamma_{2}} m_{\nu} u_{T}(t) \cdot \nabla_{T} u_{\nu}(t) d \Gamma$ at $t=0$ or $T$. For that purpose, we consider $\zeta \in H^{1}\left(\Gamma_{2}\right)$ for the solution of

$$
\zeta-\Delta_{T} \zeta=\operatorname{div}_{T} u_{T}(t) \text { on } \Gamma_{2} .
$$

As $\operatorname{div}_{T} u_{T}(t)$ belongs to $H^{-1 / 2}\left(\Gamma_{2}\right), \zeta$ belongs to $H^{3 / 2}\left(\Gamma_{2}\right)$, with the estimate

$$
\|\zeta\|_{H^{3 / 2}\left(\Gamma_{2}\right)} \leq C\left\|\operatorname{div}_{T} u_{T}(t)\right\|_{H^{-1 / 2}\left(\Gamma_{2}\right)} \leq C\left\|u_{T}(t)\right\|_{H^{1 / 2}\left(\Gamma_{2}\right)} .
$$

Note further that the variational formulation of the above problem leads to

$$
\|\zeta\|_{H^{1}\left(\Gamma_{2}\right)} \leq C\left\|u_{T}(t)\right\|_{L^{2}\left(\Gamma_{2}\right)} .
$$

Now by Green's formula and the definition of $\zeta$ we have

$$
\begin{aligned}
\int_{\Gamma_{2}} m_{\nu} u_{T}(t) \cdot \nabla_{T} u_{\nu}(t) d \Gamma & =\int_{\Gamma_{2}} u_{\nu}(t) \operatorname{div}_{T}\left(m_{\nu} u_{T}(t)\right) d \Gamma \\
& =\int_{\Gamma_{2}} u_{\nu}(t) u_{T}(t) \cdot \nabla_{T} m_{\nu} d \Gamma \\
& -\int_{\Gamma_{2}} u_{\nu}(t) m_{\nu} \zeta d \Gamma+\int_{\Gamma_{2}} u_{\nu}(t) m_{\nu} \Delta_{T} \zeta d \Gamma .
\end{aligned}
$$

Using Cauchy-Schwarz's inequality and the estimate (176), we obtain

$$
\left|\int_{\Gamma_{2}} u_{\nu}(t) \nabla_{T} m_{\nu} \cdot u_{T}(t) d \Gamma+\int_{\Gamma_{2}} u_{\nu}(t) m_{\nu} \zeta d \Gamma\right| \leq C \int_{\Gamma_{2}}|u(t)|^{2} d \Gamma .
$$

Finally, using the fact that $-\Delta_{T}$ is a nonnegative selfadjoint operator, we may write

$$
-\int_{\Gamma_{2}} u_{\nu}(t) m_{\nu} \Delta_{T} \zeta d \Gamma=\int_{\Gamma_{2}}\left(-\Delta_{T}\right)^{1 / 4}\left(u_{\nu}(t) m_{\nu}\right)\left(-\Delta_{T}\right)^{3 / 4} \zeta d \Gamma,
$$

and by Cauchy-Schwarz's inequality and the estimate (75), we get

$$
\begin{aligned}
\left|\int_{\Gamma_{2}} u_{\nu}(t) m_{\nu} \Delta_{T} \zeta d \Gamma\right| & \leq C\left\|u_{\nu}(t) m_{\nu}\right\|_{H^{1 / 2}\left(\Gamma_{2}\right)}\|\zeta\|_{H^{3 / 2}\left(\Gamma_{2}\right)} \\
& \leq C\|u\|_{H^{1 / 2}\left(\Gamma_{2}\right)}^{2} .
\end{aligned}
$$


By a standard trace theorem and the definition of the energy, we have obtained that

$$
\left|\int_{\Gamma_{2}} m_{\nu} u_{T}(t) \cdot \nabla_{T} u_{\nu}(t) d \Gamma\right| \leq C E(t)
$$

All together we have proved that

$$
\begin{aligned}
\left|\mathcal{I}_{2}\right| & \leq \theta \int_{0}^{T} \int_{\Gamma_{2}}\left(\varepsilon_{T}\left(u_{T}\right): \varepsilon_{T}\left(u_{T}\right)+\left|\varepsilon_{S}(u)\right|^{2}+\left|\partial_{\nu} u_{\nu}\right|^{2}\right) d \Gamma d t \\
& +\frac{C}{\theta} \int_{0}^{T} \int_{\Gamma_{2}}\left(|u|^{2}+\left|u^{\prime}\right|^{2}\right) d \Gamma d t+C E(0)
\end{aligned}
$$

for any $\theta \in(0,1)$.

The estimates (74) and (77) into (73) lead to

$$
\begin{aligned}
I_{5} & \leq k_{5}\left(E(0)+\int_{\Sigma_{2 T}}\left(|u|^{2}+\left|u^{\prime}\right|^{2}\right) d \Gamma d t\right) \\
& +2 \theta \int_{0}^{T} \int_{\Gamma_{2}}\left(\varepsilon_{T}\left(u_{T}\right): \varepsilon_{T}\left(u_{T}\right)+\left|\varepsilon_{S}(u)\right|^{2}+\left|\partial_{\nu} u_{\nu}\right|^{2}\right) d \Gamma d t \\
& -k \delta \int_{0}^{T} \int_{\Gamma_{2}}\left(\varepsilon_{T}(u): \varepsilon_{T}(u)+2\left|\varepsilon_{S}(u)\right|^{2}+\left|\partial_{\nu} u_{\nu}\right|^{2}\right) d \Gamma d t
\end{aligned}
$$

for any $\theta \in(0,1)$ and for some $k_{5}>0$. Choosing $\theta<k \delta / 2$, we arrive at the estimate

$$
I_{5} \leq k_{5}\left(E(0)+\int_{\Sigma_{2 T}}\left(|u|^{2}+\left|u^{\prime}\right|^{2}\right) d \Sigma\right)
$$

Owing to Lemma 5.2, we thus obtain

$$
I_{5} \leq\left(k_{5}+\frac{k_{6}}{\varepsilon}\right) E(0)+k_{5} \int_{\Sigma_{2 T}}\left|u^{\prime}\right|^{2} d \Sigma+\varepsilon \int_{0}^{T} E(t) d t,
$$

for some $k_{6}>0$.

Taking into account the definition of $\gamma_{m}$, we have

$$
I_{6} \leq \gamma_{m} \int_{0}^{T} E(t) d t
$$

All together we have shown that

$$
\begin{aligned}
2 \int_{0}^{T} E(t) d t & \leq\left(k_{1}+\frac{k_{2}+k_{6}}{\varepsilon}+k_{5}+k_{4}^{\prime}\right) E(0) \\
& +\left(\frac{k_{3}}{\varepsilon}+k_{5}+R_{0}\right)\left[\int_{\Sigma_{2 T}}\left|u^{\prime}\right|^{2} d \Sigma+\int_{Q_{T}}\left|u^{\prime}\right|^{2} d x d t\right] \\
& +\varepsilon\left(2+k_{3}+k_{4}\right) \int_{0}^{T} E(t) d t+\gamma_{m} \int_{0}^{T} E(t) d t
\end{aligned}
$$

Finally, choosing $\varepsilon \in] 0,\left(2-\gamma_{m}\right) /\left(2+k_{3}+k_{4}\right)[$ and setting

$$
C_{1}=\frac{\frac{k_{3}}{\varepsilon}+k_{5}+R_{0}}{2-\gamma_{m}-\varepsilon\left(2+k_{3}+k_{4}\right)}, C_{2}=\frac{k_{1}+\frac{k_{2}+k_{6}}{\varepsilon}+k_{5}+k_{4}^{\prime}}{2-\gamma_{m}-\varepsilon\left(2+k_{3}+k_{4}\right)},
$$


we conclude that

$$
\int_{0}^{T} E(t) \leq C_{2} E(0)+C_{1}\left(\int_{\Sigma_{2 T}}\left|u^{\prime}\right|^{2} d \Sigma+\int_{Q_{T}}\left|u^{\prime}\right|^{2} d x d t\right) .
$$

This estimate remains valid for weak solutions by a density argument.

In the formalism of Section 4, the estimate (79) means that the pair $\left(-A_{1}, \mathcal{I}_{U}\right)$ satisfies the stability estimate. We then conclude by Theorem 4.4 (see also Theorem 5.3 of [13]).

6. Examples. 1. If we assume that $f=0$, and that $g$ satisfies (5), (6), (12), (15), (16), as well as

$$
\begin{aligned}
& x \cdot g(x) \geq c_{0}|x|^{p+1}, \forall|x| \leq 1 \\
& |g(x)| \leq C_{0}|x|^{\alpha}, \forall|x| \leq 1
\end{aligned}
$$

where $c_{0}, C_{0}$ are positive constants, $\alpha \in(0,1]$ and $p \geq \alpha$. Then making the choice

$$
G(x)=x^{\frac{2}{q+1}} \text { and } q=\frac{p+1}{\alpha}-1
$$

we obtain decays similar to the ones from Theorem 2.3 of [16]. Indeed, if $p=\alpha=1$, then $\Psi^{-1}(t)=e^{-t}$ and we conclude an exponential decay. Conversely, if $p+1 \geq 2 \alpha$, then $\Psi^{-1}(t)=t^{\frac{2}{1-q}}$ and we obtain a decay of order $t^{-\frac{2 \alpha}{p+1-2 \alpha}}$.

2. Take $f=0$ and $g(\xi)=\exp \left(-\frac{1}{|\xi|^{2 p}}\right) \frac{\xi}{|\xi|^{2}}$ for $|\xi|$ small enough and for $p>0$. Then by Example 2.4 of [3], (16) holds with

$$
G(x)=\frac{C}{|\log x|^{\frac{1}{p}}}
$$

and some constant $C>0$. In the setting of Theorem 2.2 we will get the decay

$$
E(t) \leq \frac{C}{|\log t|^{\frac{1}{p}}}
$$

since $\Psi^{-1}$ is bounded from below.

In a similar manner taking $f=0$ and $g(\xi)=\exp \left(-\exp \left(1 /|\xi|^{2 p}\right)\right) \frac{\xi}{|\xi|^{2}}$ for $|\xi|$ small enough and some $p>0$. Then by Example 2.5 of [3] (16) holds with

$$
G(x)=\frac{C}{\left.|\log | \log x\right|^{\frac{1}{p}}}
$$

and some constant $C>0$. In the setting of Theorem 2.2 we will get the decay

$$
E(t) \leq \frac{C}{\left.|\log | \log t\right|^{\frac{1}{p}}}
$$




\section{REFERENCES}

[1] BEY, R.; HEMINNA, A.; LOHEAC, J.-P., Boundary stabilization of a linear elastodynamic system with variable coefficients, E.J.D.E. 2001 (2001), Num. 78, 1-23. MR.1872057(2002h:93089)

[2] BROSSARD, R.; LOHEAC, J.-P., Stabilisation frontière du système élastodynamique dans un polygone plan, C. R. Acad. Sci. Math. 338 (2004), 213-218. MR2038326 (2004k:93068)

[3] ELLER, M.; LAGNESE, J.E.; NICAISE, S., Decay rates for solutions of a Maxwell system with nonlinear boundary damping, Comp. and Appl. Math., 21 (2002), 135-165. MR2009950 (2004i:78006)

[4] GUESMIA, A., Existence globale and stabilisation frontière non linéaire d'un système d'élasticité, Portugaliae Mathematica, 56 (1999), 361-379. MR1716059 (2001k:74073)

[5] HANSEN, S.W., Exponential energy decay in a linear thermoelastic rod, J. Math. Anal. Appl. 167 (1992), 429-442. MR:1168599 (93f:35229)

[6] HEMINNA, A.; NICAISE, S.; SENE A., Stabilisation d'un système de la thermoélasticité avec feebacks non linéaires, C.R. Acad. Sci. Paris, série I Math. 339 (2004), 561-566. MR2111352

[7] HORN, M.A., Implications of sharp trace regularity results on boundary stabilization of the system of linear elasticity, J. Math. Anal. Appl. 223 (1998), 126-50. MR1627344 (99c:93059)

[8] KOMORNIK, V., Exact Controllability And Stabilization, The Multiplier Method, RMA 36, Masson, 1994. MR1359765 (96m:93003)

[9] LEMRABET, K., Étude de divers problèmes aux limites de Ventcel d'origine physique ou mécanique dans des domaines non réguliers, Thesis, U.S.T.H.B., Alger, 1987.

[10] NARUKAWA, K., Boundary value control of thermoelastic systems, Hiroshima Math. J. 13 (1983), 227-272. MR 0707182 (85c:93021)

[11] NICAISE, S.; SENE, A., Stabilisation non linéaire d'un système de la thermoélasticité isotrope, Preprint MACS 03-12, 2003.

[12] NICAISE, S., Stability and controllability of the electromagneto-elastic system, Portugaliae Mathematica 60 (2003), 37-70. MR.1961551(2003m:93008)

[13] NICAISE, S., Stability and controllability of and abstract evolution equation of hyperbolic type and concrete applications, Rendiconti di Matematica, Serie VII, 23 (2003), 83-116. MR2044992 (2005a:93024)

[14] LEBEAU, G.; ZUAZUA, E., Decay rates for the three-dimensional linear system of thermoelasticity, Arch. Rat. Mech. Anal. 148 (1999) 179-231. MR:1716306 (2000h:35154)

[15] LIU, W.J., Partial exact controllability and exponential stability in higher-dimensional linear thermoelasticity, ESAIM COCV 3 (1998), 23-48. MR1610226 (99i:93056a)

[16] LIU, W.J.; ZUAZUA.E., Uniform stabilization of the higher-dimensional system of thermoelasticity with a nonlinear boundary feedback, Quarterly of Applied Mathematics 59 (2001), 269-314. MR 1828455 (2002d:74018)

[17] LIU, Z.; ZHENG, S., Exponential stability of the semigroup associated with a thermoelastic system, Quart. Appl. Math. 51 (1993), 535-545. MR1233528 (95c:35247)

[18] MUÑOZ RIVERA, J.E., Decomposition of the displacement vector field and decay rates in linear thermoelasticity, SIAM J. Math. Anal. 24 (1993), 390-406. MR1205533 (94a:73007)

[19] MUÑOZ RIVERA, J.E., Asymptotic behaviour in n-dimensional thermoelasticity, Appl. Math. Lett. 10 (1997), 47-53. MR1471316 (98j:73016)

[20] OLIVEIRA, L.A.F., Exponential decay in thermoelasticity, Commun. Appl. Anal. 1 (1997), 113-118. MR1453945 (98d:73011)

[21] SHOWALTER, R.E., Monotone Operators in Banach Space and Nonlinear Partial Differential Equations, Math. Surveys and Monographs, 49, AMS, 1997. MR.1422252 (98c:47076)

[22] VALID, R., La mécanique des milieux continus et le calcul des structures, Eyrolles, Paris, 1977. 